\title{
Multi-stage method to identify structural damage using multiple damage location assurance criterion and an improved differential evolution algorithm
}

\author{
Chunli Wu ${ }^{1}$, Hanbing Liu ${ }^{1}$, Xuxi Qin ${ }^{1}{ }^{*}$, Guojin Tan ${ }^{1}$, Zhengwei Gu ${ }^{1}$ \\ ${ }^{1}$ College of Transportation, Jilin University, Changchun 130021, PR China \\ E-mail: clwu@jlu.edu.cn(Wu),liuhb_57@126.com(Liu),qinxx29@sina.com(Qin),tgj@jlu.edu.cn(Tan), \\ zhengweigu123@sina.com(Gu)
}

Received 10 February 2017

Accepted 4 July 2017

\begin{abstract}
For the damage identification technique of civil structures, the reduction of the computational cost for methods based on the optimization algorithms is the most crucial step. In this study, a fast multi-stage method is developed that uses the multiple damage location assurance criterion and an improved differential evolution algorithm. In the new method, the suitable damage range is selected in different stages to reduce the computational cost of structural analysis. Five mutation operators are analysized not only in the basic differential evolution algorithm but also in the improved one. A new adaptive scaling factor with a segmented function is proposed which can operate the decay rate to avoid the premature phenomenon. The results of the study show that the precise locations and extents of structural damage are successfully realized. It is also shown that the multi-stage method using the improved differential evolution algorithm is substantially faster as compared to the basic one and can be used as an efficient and powerful measure of structural damage identification.
\end{abstract}

Keywords: damage identification, multiple damage location assurance criterion, improved differential evolution algorithm, multi-stage method, adaptive scaling factor.

\section{Introduction}

Civil engineering structures have always been susceptible to various kinds of damage during their service life due to environmental, operational and human-induced factors. Therefore, damage identification has been essential issues for determining safety reliability and remaining lifetime of structures. Although a mass of damage identification methods are proposed in the many literatures in the past several decades, the vibration-based structural damage detection has been one of the best hot issues of research. The basic idea of the vibration-based damage identification approaches is that the changes in the physical properties (mass, stiffness and daming) due to damage will be reflected in modal proprerties (frequencies, mode shapes and modal damping). Hence, the damage identification as an inverse problem can be realized by detecting the changes of modal properties before and after damage by using the finite element model and given limited modal data measured from a real structure. Reviews of these methods can be found in Refs. 1-5. More study efforts had been implemented based on frequency change-based method ${ }^{6,7}$, mode shape change-based method ${ }^{8-10}$, frequency and mode shape change-based method ${ }^{11}$, mode shape curvature ${ }^{12,13}$, modal strain enery ${ }^{14,15}$, flexibility based approach 16,17 , and frequency response function ${ }^{18-19}$.

Intelligence optimal algorithms have been widely employed for structural damage detection. The main idea of optimal algorithms is transforming the damage

* Corresponding author. 
identification problem into an optimization problem in which the objective function composed by the alteration between the FEM and measured data is usually minimized or maxmized. So far, an increasing number of optimal algorithms such as neural network (NE) ${ }^{20,21}$, Genetic algorithms $(\mathrm{GA})^{22,}{ }^{23}$, particle swarm optimization (PSO) ${ }^{24,25}$, artificial bee colony $(\mathrm{ABC})^{26,}$ ${ }^{27}$, differential evolution $(\mathrm{DE})^{28-30}$ and wavelet analysis 31,32 etc are widely used in determining the location and extent of structural damage. However, the expensive computational cost and the invalid for dealing with large civil engineering structures of the optimization algorithms still need to be addressed eagerly.

Phased approaches such as two-step and multi-stage methods using in detecting both the damage location and extent have been employed to reduce the expensive computational cost. Two-stage method is the most common, in which the possible damage sites are determined in the first stage using certain modal indicators, for examples, the modal strain energy ${ }^{28}$, evidence theory combined with natural frequencies and mode shapes ${ }^{22}$, modal residual vector ${ }^{29}$, modal strain energy and frequency integrated with Bayesian theory ${ }^{23}$, and the damage extents are detected in the next stage by the use of an improved $\mathrm{DE}^{28}$, micro-search $\mathrm{GA}^{22}$, $\mathrm{GA}^{29}$, immune $\mathrm{GA}^{23}$ with respect to the examples of the first stage. Further more, the multi-stage methods are proposed in few researches. Grande ${ }^{33}$ proposed a multistage approach with the mode shapes assumed as primary sources and local decisions based on a flexibility method. Nevertheless, the high computational cost of detecting damage locations, especially the damage extents of the large structures still is an urgent task to resolve for damage identification technique based on optimal algorithms.

This paper makes an effort to further reduce the computational cost of detecting damage sites and extents of civil engineering structures by proposing a multi-stage method with multiple damage location assurance criterion (MDLAC) and an improved differential evolution (IDE) algorithm. Unlike the other researches, in this paper, MDLAC is regarded as the objective function of optimal algorithm and that differential evolution algorithm is as the optimal tool in each stage. For the present multi-stage method, the small damage range and filtering threshold are used in the earlier stage to reduce the design variables of the optimization problem for the later stages. Moreover, this paper analyzes and compares five mutation operators and different scaling factors, and proposes a new adaptive scaling factor with a segmented function in order to find the more efficient mutation operator. The numerical examples consider a continous beam and a square composite plate, as well as the effect of noise on the accuracy of the present procedure is investigated. The results of the study show that regardless of the effect of noise, the precise locations and extents of structural damage are successfully realized, especially for the computational cost.

This study is organized as follows: In Section 2, damage indexes based on the natural frequency, mode shape and both the frequency and mode shape are presented, respectively. Section 3 gives the introduction of the basic differential evolution algorithm and the improved differential evolution algorithm in terms of the scale factor $F$. In Sections 4, the multi-stage damage identification method is proposed including the individual, damage range and filtering threshold. In Sections 5, the performance of the multi-stage damage identification method is demonstrated with a continous beam and a square composite plate.

\section{Damage indices of multiple damage locations}

\subsection{Damage index based on natural frequency}

MDLAC is a powerful tool to identify the location and extent of damage existing in structures, which is performed by considering the correlation between the modal parameters monitored in practical structures and parameters calculated by Finite Element Analysis (FEA) 34-36.

The natural frequency is first used, and afterwards the mode shape is introduced in the MDLAC. When only the natural frequency is considered, MDLAC_f can be expressed as

$$
\operatorname{MDLAC} \_f(X)=\frac{\left|\Delta F^{\mathrm{T}} \cdot \delta F(X)\right|^{2}}{\left(\Delta F^{\mathrm{T}} \cdot \Delta F\right)\left(\delta F^{\mathrm{T}}(X) \cdot \delta F(X)\right)}
$$

where $\Delta F$ is the shift vector of the experimental frequencies before and after the damage occur, and $\delta F(X)$ is the change vector between the undamaged frequencies and theoretical frequencies calculated by FEA at the $n^{\text {th }}$ position. $\Delta F$ and $\delta F(X)$ can be 
represented using the following expressions, respectively.

$$
\begin{gathered}
\Delta F=\frac{F_{h}-F_{d}}{F_{h}} \\
\delta F(X)=\frac{F_{h}-F(X)}{F_{h}}
\end{gathered}
$$

where $F_{h}$ and $F_{d}$ are the natural frequencies before and after the damage occur, respectively. $F(X)$ is the theoretical frequency from theoretical model, in which $X^{\mathrm{T}}=\left\{x_{1}, x_{2}, \cdots, x_{D}\right\}$ is a variable vector of all $D$ elements as well as implying the extent of damage. For example, $x_{6}=0.05$ denotes that the damage occurs at the 6 th element and the extent of damage is $5 \%$.

The values of MDLAC_f vary between zero and one. A value of zero denotes no correlation between the measured and theoretical frequency shift, and a value of one indicates exact match, i.e. $F(X)=F_{d}$. This shows that the highest value of MDLAC_f is optimal for determining the location and extent of damage.

\subsection{Damage index based on mode shape}

The processes of damage detection using the MDLAC based on natural frequency are demonstrated commonly by the symmetrical structure, for example, a cantilever beam in Refs.34 and 35. However, only the MDLAC with respect to the frequency fails to asymmetric structures, especially such as civil engineering structures. As a consequence, the mode shape owing to the excellent local sensitivity for damage is introduced to the damage index of MDLAC. The MDLAC_ $\Phi$ is given by

$$
\operatorname{MDLAC} \Phi(X)=\frac{\left|\Delta \Phi^{\mathrm{T}} \cdot \delta \Phi(X)\right|^{2}}{\left(\Delta \Phi^{\mathrm{T}} \cdot \Delta \Phi\right)\left(\delta \Phi^{\mathrm{T}}(X) \cdot \delta \Phi(X)\right)}
$$

where $\Delta \Phi$ is the shift vector of the mode shapes before and after the damage occur, and $\delta \Phi(X)$ is the change vector between the undamaged and theoretical mode shapes. $\Delta \Phi$ and $\delta \Phi(X)$ can be expressed as

$$
\begin{gathered}
\Delta \Phi=\frac{\Phi_{h}-\Phi_{d}}{\Phi_{h}} \\
\delta \Phi(X)=\frac{\Phi_{h}-\Phi(X)}{\Phi_{h}}
\end{gathered}
$$

where $\Phi_{h}$ and $\Phi_{d}$ are the mode shapes before and after the damage occurs, respectively. $\Phi(X)$ is the theoretical mode shape.

Similar to considering the frequency, the values of MDLAC_ $\Phi$ vary between zero and one. A value of zero denotes no correlation and one indicates exact match, i.e. $\Phi(X)=\Phi_{d}$. Therefore, the aim of damage identification focuses on maximizing the value of MDLAC_ $\Phi$ by finding the vector $X$ contained the degree of damage.

\subsection{Damage index based on both frequency and mode shape}

A MDLAC_f $+\Phi$ is proposed based on both frequencies and mode shapes, which can address well both symmetrical and asymmetrical structures ${ }^{35,36}$. This combined index is defined by

$$
\begin{aligned}
& \operatorname{MDLAC} \mathrm{f}_{+}+\Phi(X) \\
& =\alpha \cdot \operatorname{MDLAC}_{-} \mathrm{f}+\beta \cdot \mathrm{MDLAC}_{-} \Phi \\
& =\alpha \cdot \frac{\left|\Delta F^{\mathrm{T}} \cdot \delta F(X)\right|^{2}}{\left(\Delta F^{\mathrm{T}} \cdot \Delta F\right)\left(\delta F^{\mathrm{T}}(X) \cdot \delta F(X)\right)} \\
& +\beta \cdot \frac{\left|\Delta \Phi^{\mathrm{T}} \cdot \delta \Phi(X)\right|^{2}}{\left(\Delta \Phi^{\mathrm{T}} \cdot \Delta \Phi\right)\left(\delta \Phi^{\mathrm{T}}(X) \cdot \delta \Phi(X)\right)}
\end{aligned}
$$

where $\alpha$ and $\beta$ are the scale factors and $\alpha+\beta=1$, the magnitude of them can be determined by the experimental or practical structures.

Now, the problem of damage identification can be solved by searching the vector $X$ to maximize the index MDLAC $\mathrm{f}+\Phi$. An optimization algorithm is required to solve this issue, and the optimization process can be described as follows.

$$
\begin{aligned}
& \text { Find } \quad X^{\mathrm{T}}=\left\{x_{1}, x_{2}, \cdots, x_{n}\right\} \\
& \text { Maximize } \omega+\Phi(X)=\text { MDLAC_f }+\Phi(X)
\end{aligned}
$$

\section{Differential evolution algorithm}

\subsection{Basic differential evolution algorithm}

Differential Evolution (DE) Algorithm is a global search algorithm inspired by Darwin's survival-of-thefittest theory, which can also obtain the optimal solution by three main operators including the mutation, cross and selection ${ }^{28-30}$. Provided that the individual of the $G^{\text {th }}$ generation is $X_{i, G}$ and $i=1,2, \cdots, N P$, in which $N P$ is the population size, some basic concepts and operators of a DE can be introduced as follows.

\subsubsection{Coding}

An essential characteristic of a DE is the coding of the individuals that describe the optimization problem. The real coding can be written as $X_{i, G}=\left(x_{1}, x_{2}, \cdots, x_{D}\right)$. 


\subsubsection{Initial population}

The following expression is used to initialize population.

$$
x_{j}=x_{j}^{L}+\operatorname{rand} \cdot\left(x_{j}^{U}-x_{j}^{L}\right), j=1,2, \cdots, D
$$

where rand varies from zero to one randomly. $x_{j}^{U}$ and $x_{j}^{L}$ are the upper and lower limit values of the individual, respectively.

\subsubsection{Mutation}

The mutation operator is a main step to generate the new individuals in the DE. At the same time, this operator is the biggest difference between the DE and GA or other algorithms. The middle individuals after the process of the mutation are $Y_{i, G}$, which can be obtained by the linear combinations as follows. rand1:

$$
Y_{i, G}=X_{r 1, G}+F \cdot\left(X_{r 2, G}-X_{r 3, G}\right)
$$

rand2:

$$
\begin{aligned}
Y_{i, G} & =X_{r 5, G}+F \cdot\left(X_{r 1, G}+X_{r 2, G}\right. \\
& \left.-X_{r 3, G}-X_{r 4, G}\right)
\end{aligned}
$$

best1:

best2:

$$
Y_{i, G}=X_{b e s t, G}+F \cdot\left(X_{r 1, G}-X_{r 2, G}\right)
$$

$$
\begin{aligned}
Y_{i, G} & =X_{b e s t, G}+F \cdot\left(X_{r 1, G}+X_{r 2, G}\right. \\
& \left.-X_{r 3, G}-X_{r 4, G}\right)
\end{aligned}
$$

current to rand:

$$
\begin{aligned}
Y_{i, G} & =X_{i, G}+F \cdot\left(X_{r 1, G}-X_{i, G}\right) \\
& -F \cdot\left(X_{r 2, G}-X_{r 3, G}\right)
\end{aligned}
$$

current to best:

$$
\begin{aligned}
Y_{i, G} & =X_{i, G}+F \cdot\left(X_{\text {best }, G}-X_{i, G}\right) \\
& -F \cdot\left(X_{r 1, G}-X_{r 2, G}\right)
\end{aligned}
$$

where $X_{b e s t, G}$ is the best individual. $X_{r 1, G}, X_{r 2, G}$, $X_{r 3, G}, X_{r 4, G}$ and $X_{r 5, G}$ are five different individuals selected randomly before the mutation operation, $r 1, r 2, r 3, r 4, r 5 \in\{1,2, \cdots, N P\} \quad$ and $r 1 \neq r 2 \neq r 3 \neq r 4 \neq r 5 \neq i . F$ is called the scaling factor controlling the mutation scale, which is generally restricted in $(0,1]$.

It should be noted that $F$ is an essential parameter in DE because it improves the diversity of population. A small $F$ will lead to extensive exploitation in the search space, whereas a large $F$ will lead to over exploration and low convergence speed.

\subsubsection{Crossover}

The crossover operator can further promote the diversity of population by hybridizing the individuals $Y_{i, G}$ and
$X_{i, G}$. Assuming that the new candidate solutions are $U_{i, G}$, and it can be written as $U_{i, G}=\left(u_{1 i, G}, u_{2 i, G}, \cdots, u_{D i, G}\right)$, in which $i=1,2, \cdots, N P$ and $j=1,2, \cdots, D$. The new individual by the crossover operation $u_{j i, G}$ can be expressed in the form:

$$
u_{j i, G}=\left\{\begin{array}{cc}
y_{j i, G} & \text { if } \operatorname{randb}(j) \leq C R \text { or } j=\operatorname{rnbr}(i), \\
x_{j i, G} & \text { else. }
\end{array}\right.
$$

where $C R$ is the crossover rate, which is the another control parameter in the DE because it determines the probability of the middle individuals to replace the candidate individuals $U_{i, G}$. Namely, the higher is the $C R$, the faster the update speed is. $\operatorname{rand} b(j) \in[0,1]$ is a random number of uniform distribution, and $\operatorname{rnbr}(i)$ is a random integer varying between zero and one, both of which guarantee that the candidate individual $u_{j i, G}$ can get a variable from the middle individual $y_{j i, G}$ at least.

\subsubsection{Selection}

The selection operator is executed by comparing the fitness function values between the individuals $U_{i, G}$ and $X_{i, G}$. The process can be expressed as

$$
X_{i, G+1}=\left\{\begin{array}{cc}
U_{i, G} & f f\left(U_{i, G}\right)<f\left(X_{i, G}\right), \\
X_{i, G} & \text { else. }
\end{array}\right.
$$

It can be shown from Eq. (17) that the selection operator is after the mutation and crossover operators in the DE algorithm, which is different from the GA. It is better that the parent individuals competing with the new candidate individuals one by one generate the new individuals on the equal opportunity.

\subsection{Improved differential evolution algorithm}

Many researches denote that the value of the scale factor $F$ should be large in the initial stage of the algorithm such that the algorithm can maintain the diversities of the individuals, search the global optimum value and speed up the convergence speed of the optimal solution so as to avoid the premature phenomenon. While in a later stage of the algorithm, the speed of the scale factor $F$ should be decreased, which should make it easy to search for the local optimum value $^{37,38}$. The dynamic adaptive adjustment equation for the scale factor $F$ is shown as follows.

$$
F(t)=\frac{F_{\min }}{1+\left(\frac{F_{\min }}{F_{\max }}-1\right) \cdot e^{-\alpha t}}
$$


where, $t$ is the iteration, $F_{\max }$ and $F_{\min }$ are the maximum value and minimum value of the scaling factor, respectively. To maintain the balance between the diversity and the convergence, the value of $F$ is $[0.5$, 1]. $\alpha$ is a factor to determine the initial decay rate of the curve, the range of $\alpha$ is $[0,+\infty]$, in this paper, the value of $\alpha$ is 0.001 .

Moreover, in Ref. 39, the value of $F$ is generated for each individual from a normal distribution $F \in \mathrm{N}(0.5$, $0.3)$, while the value is determined as $F \in \mathrm{N}(0,1)$ in Ref. 40.

In the present IDE, the segmented function is utilized as the adaptive scaling factor provided in Eq. (19).

$$
F(t)=\left\{\begin{array}{cc}
F=F_{\max } & f_{\text {best }}<f_{1} \\
F=k \cdot f_{\text {best }} & f_{1}<f_{\text {best }}<f_{2} \\
F=F_{\text {min }} & f_{\text {best }}>f_{2}
\end{array}\right.
$$

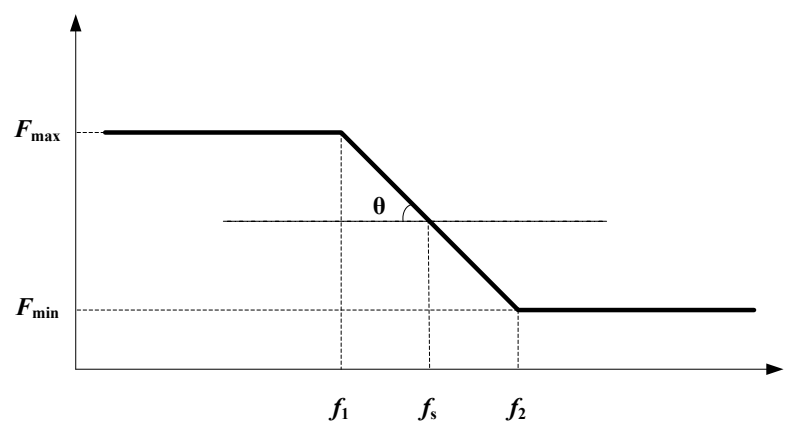

Fig. 1. Scaling factor using segmented function

where, as shown in Fig. $1, F_{\max }$ and $F_{\min }$ are the maximum value and minimum value of the scaling factor, respectively. $f_{\text {best }}$ is the fitness value of the best individual calculated by the MDLAC in each iteration. $k=\tan \theta \quad$ is the straight slope. $f_{1}=f_{s}-\left(F_{\max }-F_{\min }\right) / 2 k$ is the start dot and $f_{2}=f_{s}+\left(F_{\max }-F_{\min }\right) / 2 k$ is the end dot of changing region for fitness value. $f_{s}$ is the mid value of the fitness function in the changing region. In each iteration of optimization, the scaling factor $F$ can be determined when the parameters $F_{\max }, F_{\min }, \theta$ and $f_{s}$ are given.

\section{Multi-stage damage identification}

In this paper, the IDE algorithms with different mutation strategies and different scaling factors are proposed to identify the damage sites and extents in the structures. Some important control parameters are required in the process of implementing the multi-stage damage identification, such as the individual, damage range and filtering threshold, which are introduced in the following.

\subsection{Individual}

The individual can be expressed as $X^{T}=\left\{x_{1}, x_{2}, \cdots, x_{i}, \cdots, x_{D}\right\}$, in which $x_{i}$ represents the damage of the structures mentioned earlier. In most studies of damage detection, the damage is represented by the stiffness reduction because the damage of structural elements must lead to the stiffness descending. Hence, the damage is simulated by a reduction in the bending stiffness as

$$
x_{i}=\left(E I-E I_{i}\right) / E I, i=1, \cdots, D
$$

where $E I$ and $E I_{i}$ are the initial and damaged bending stiffness, respectively.

In this paper, we suppose the inertia moment $I$ is constant and the damage is caused by the descending of elastic modulus $E$.

\subsection{Damage range}

In the present damage identification algorithm, damage range is a crucial parameter to quickly identify the damage location and extent using the present multistage method. The damage value $R$ of the variable $x_{i}$ varies from zero to one. A value of zero means no damage and inversely a value of one denotes that the element in structures is totally damaged. A larger $R$ will lead to a slow convergence speed and excessive iterations, while a smaller $R$ will lead to leave out the optimal solutions (damaged elements). Therefore, $R$ is a parameter which has a larger effect on the calculated amount and time of identifying the damage using the DE. In this paper, we suppose that the range of $R$ value is less than and equal to 0.6 (the extent of damage is $60 \%$ ). In fact, it is more reasonable because the occurrence of too much damage is a small probability event in actual structures. However, the calculated amount of the procedure is still considerably large even though $R \in[0,0.6]$ due to the complicated structural analysis. Moreover, more elements divided in the model further increase the iterations from the other hand. In view of this, a multi-stage method is proposed to reduce the calculated amount and time of the search by controlling the range of $R$. For example, in Fig. 2, the first stage is to determine the range of $R=[0,0.1]$ and then $R=[0,0.6]$ in the second stage. In this way, in the 
first step, the running time is reduced due to the small damage range even though all elements involved in calculation. Consequently, a great amount of undamaged elements are filtering out and the locations of suspected damage elements are retained. Thus, a low calculated amount occurs because only the retained elements take part in the computation process of the second step even if the damage range is enlarged.

\subsection{Filtering threshold}

The filtering threshold $\rho$ is a critical value to filter the undamaged elements and then retain the suspected damaged elements. A small $\rho$ will lead to extensive suspected damaged elements retained and thus lose the

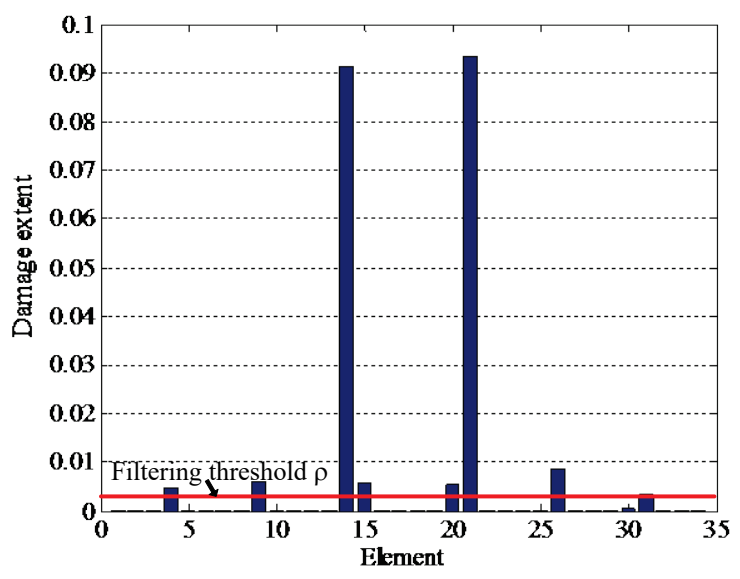

a multi-stage meaning, whereas a large $\rho$ will lead to miss the damage elements. Fig. 2 shows an example of the two-step method to identify structural damage using the frequency MDLAC_f. In this example, damage occurs in elements 9 and 14 with 5\% and $40 \%$ stiffness reduction, respectively. When the filtering threshold is selected as $\rho=0.0027$ shown in Fig. 2 (a), only eight elements $(4,9,14,15,20,21,26$ and 31) are left as the suspected elements. Thereby, the relatively accurate damage results are quickly detected as the damages of element 9 with $4.98 \%$ and element 14 with $42 \%$ without the need for further filtering stage (Fig. 2 (b)).

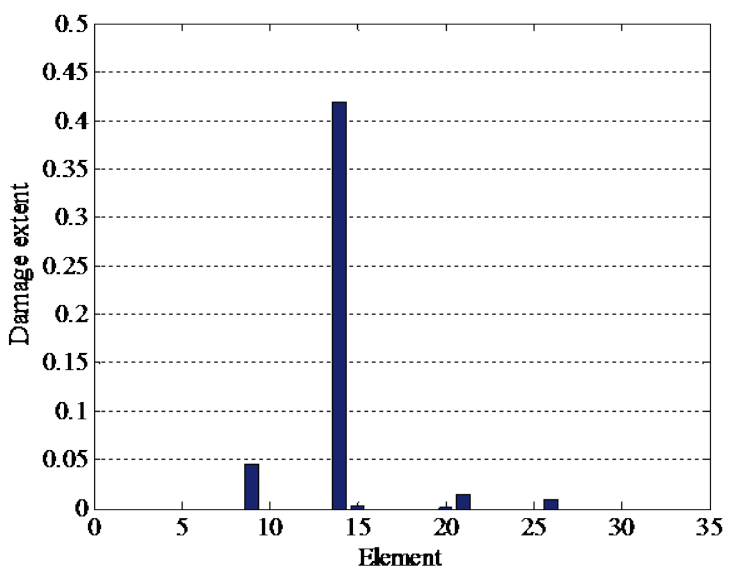

b

Fig. 2. An example of the multi-step method to identify structural damage. (a) the first stage; (b) the second stage.

The filtering threshold $\rho$ can be expressed with the subscript $\rho_{1}$ and $\rho_{2}$ in the different stages of damage identification which can be defined by computing the average value of damage individuals as

$$
\begin{aligned}
& \rho_{1}=\frac{1}{D} \sum_{i=1}^{D} x_{i} \\
& \rho_{2}=\frac{1}{m} \sum_{i=1}^{m} x_{i}
\end{aligned}
$$

where, $\rho_{1}$ is the average value of all damage individuals which is used in the second stage of the presented multi-stage method. $\rho_{2}$ is the average value of the first $m$ damage individuals when all individuals are sorted according to the descending order of damage extents which is more larger than $\rho_{1}$. Morover, it is used in the latter stages further to filter the damage elements. $m$ is selected as the quarter of all element amount in this paper.

\subsection{Multi-stage damage identification}

The damage location and extent of the structure are detected by the presented damage identification method. The flow chart of multi-stage method with MDLAC and DE or IDE is shown in Fig. 3. 


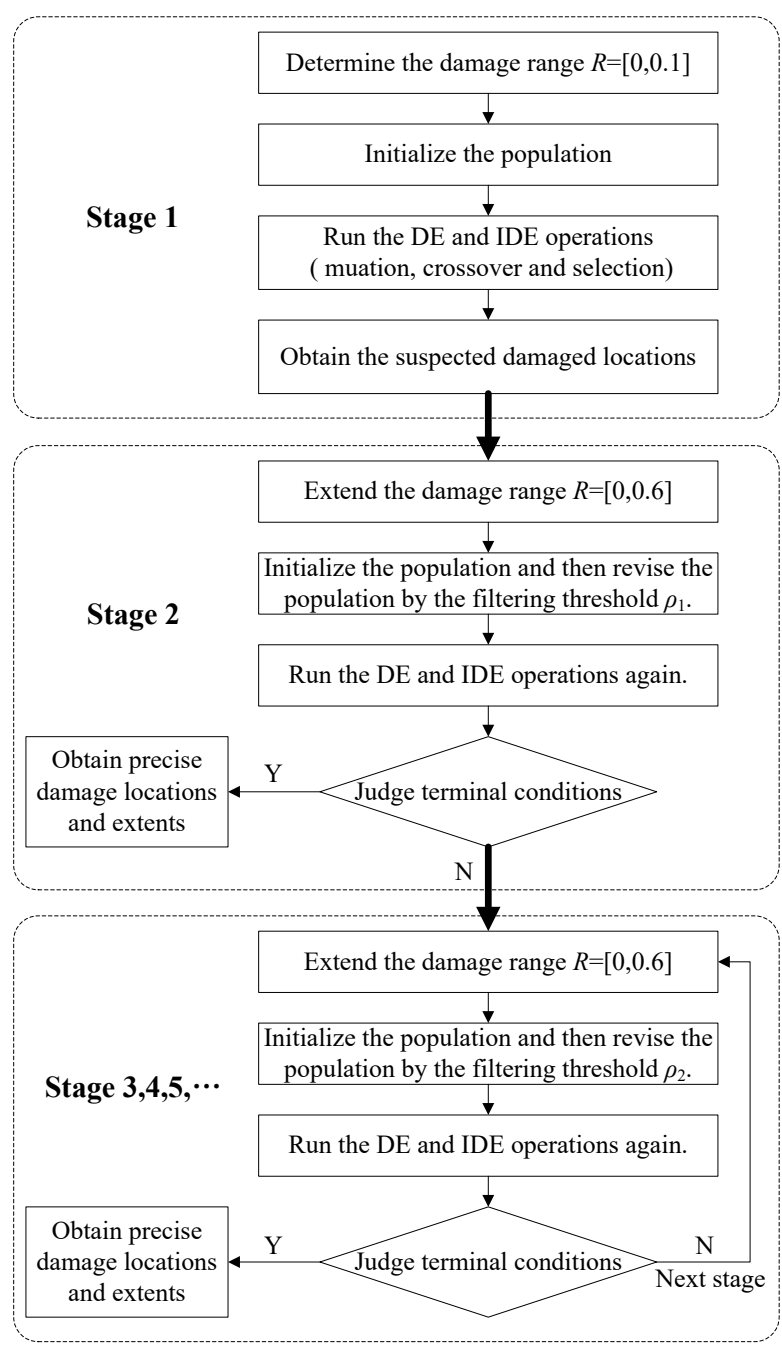

Fig. 3. Flow chart of presented damage identification method.

\section{Numerical studies}

In this section, we present a numerical example of the continuous beam to verify the reliability of the proposed damage identification technique using the MDLAC and the IDE algorithm. Some control parameters in the DE and IDE are shown in Table 1.

\subsection{A continuous beam}

\subsubsection{Model of damage}

The first numerical example considered is a continuous beam with two spans shown in Fig. 4. The values of material and geometric properties for the beam are also given in the figure. The beam is divided into 34 equal length elements. The beam is excited by random dynamic loading which is stochastic in nature. A local damage is assumed by reducing the stiffness of element 9 by $5 \%$ and element 14 by $40 \%$.

\subsubsection{Damage identification}

In the whole process of damage identification, the damage index MDLAC with frequency and mode shape for all elements of the beam is computed by modal analysis of the FEM. Two cases without noise and with noise $3 \%$ and five muation strategies are considered respectively to verify the performance of damage identification. Both in the cases of noise-free and noise, the first ten frequencies and the fist three mode shapes are used to compute the objective damage index MDLAC. The individual can be denoted by $X^{\mathrm{T}}=\left\{x_{1}, x_{2}, \cdots, x_{i}, \cdots, x_{D}\right\}(i=1, \cdots, D)$, which are the damage extents of $D$ suspected elements. Particularly, for this example, $X^{\mathrm{T}}=\left\{x_{1}, x_{2}\right\}$ in which $x_{1}$ and $x_{2}$ are the damage extents of the 9 th and 14th elements, respectively. It should be noted that the statistical results are the average solutions of ten times precedure and the average number of structural analysis (NSA).

1. Case of noise free

In the case of noise free, the damage location and extent of damage elements are implemented by the comparison with the basic DE and the present multi-stage DE and IDE. The statistical results for five kinds of mutation strategies (rand1, rand2, best1, best2, current to rand (c t r)) are shown in Fig. 5 and Table 2. In these figures, the $\mathrm{X}, \mathrm{Y}$ and $\mathrm{Z}$ axes are the number of elements, five kinds of mutation strategies and damage extent (1-MDLAC), respectively. In the basic DE, the single stop criterion is defined by the objective function $f=0.99998$. While in the present DE and IDE, the double criteria in the different stages are set as the maximum iterations $T_{1}=5000, T_{2,3, \cdots}=1000$, and objective functions $f_{1}=0.995, \quad f_{2,3, \cdots}=0.99998 \quad$, respectively. In addition, in the IDE, the proposed segmented function is used to improve the scaling factor $F$ in the Stage 1, and the normal distribution $F \in \mathrm{N}(0.5,0.3)$ is utilized in the subsequent stages. For the scaling factor of segmented function, some controlled parameters in Eq. (19) are set as: $F_{\max }=0.9, F_{\min }=0.5, f_{s}=0.8$, and $\theta=80^{\circ}$.

It can be seen that for the case of noise free from Fig. 5 and Table 2, the precise locations and extents of damaged elements can be detected by both the basic DE and the present DE and IDE under the computation of two stages. However, for the computaional cost, the 
NSA of the basic DE is more than the present multi_stage DE and IDE, while the IDE shows the preferable performance than the present DE due the less NSA. For different muataion strategies, the rand1 and rand 2 are stable relatively, and that the best 1 and best 2 emerge the strong ability of computation, especially for the best 1 with the least NSA.

2. Case of noise level $3 \%$

In the case of measurement noise, the basic DE and the present mult-stage DE and IDE are used to detect the damage locations and extents. The statistical results for five kinds of mutation strategies are shown in Fig. 6 and Table 3. In these figures, the $\mathrm{X}, \mathrm{Y}$ and $\mathrm{Z}$ axes are the number of elements, two kinds of mutation strategies and damage extent (1-MDLAC), respectively. In addition, the stop criteria in the multistage process need to be provided. They are: the maximum iterations $T_{1}=2000, T_{2,3, \cdots}=1000$, and objective functions $f_{1}=0.9048, f_{2,3, \cdots}=0.92$, respectively. It should be noted that the scaling facors and the relevent parameters used in this case are same with the case of noise free in different stages of the proposed multi-stage method.

Table 1 Parameters of the DE and IDE using the multi-stage method

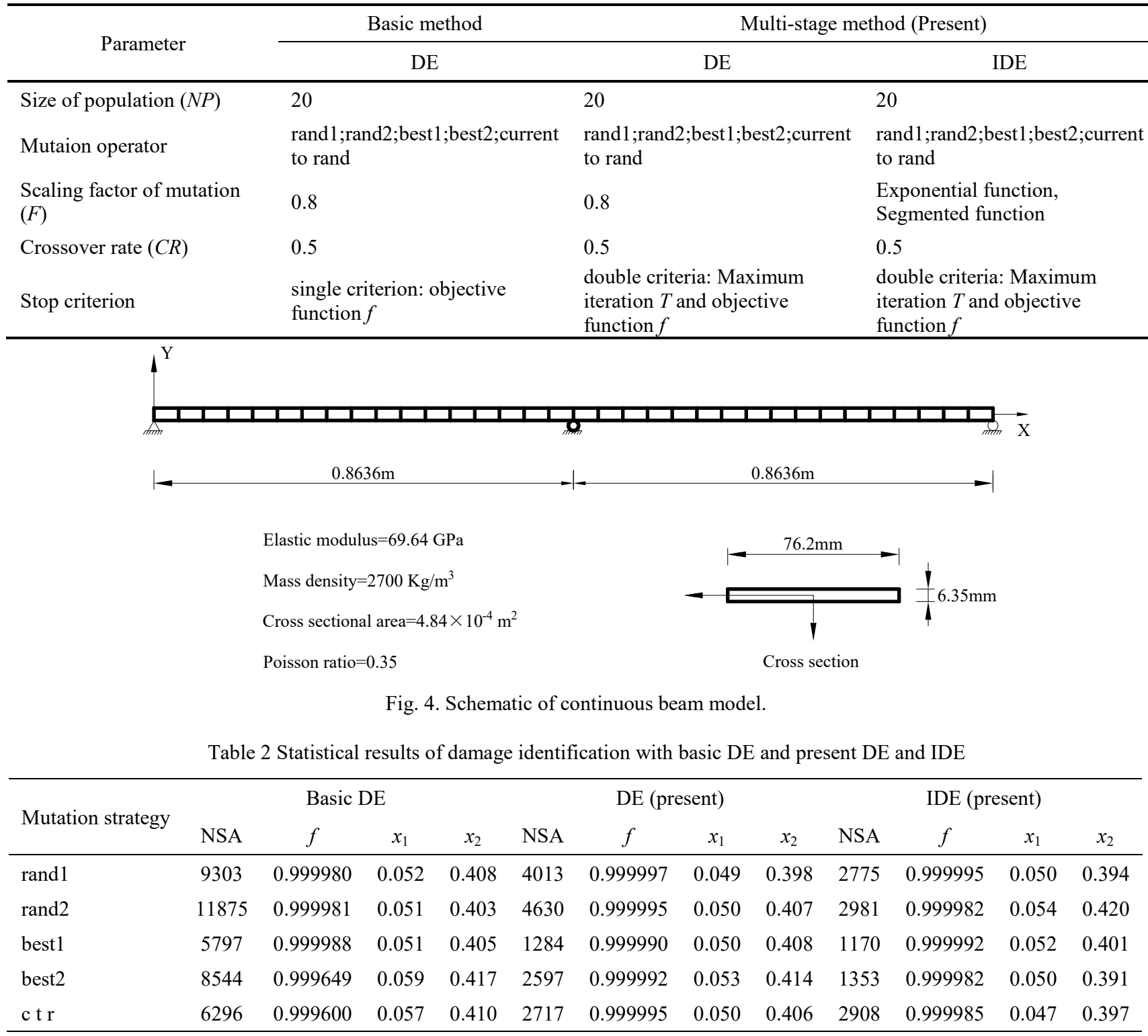

where, $\mathrm{NSA}=$ average number of structural analysis; $f=$ the value of objective function $x_{1}=$ damage extent of element $9 ; x_{2}=$ damage extent of element 14 . 

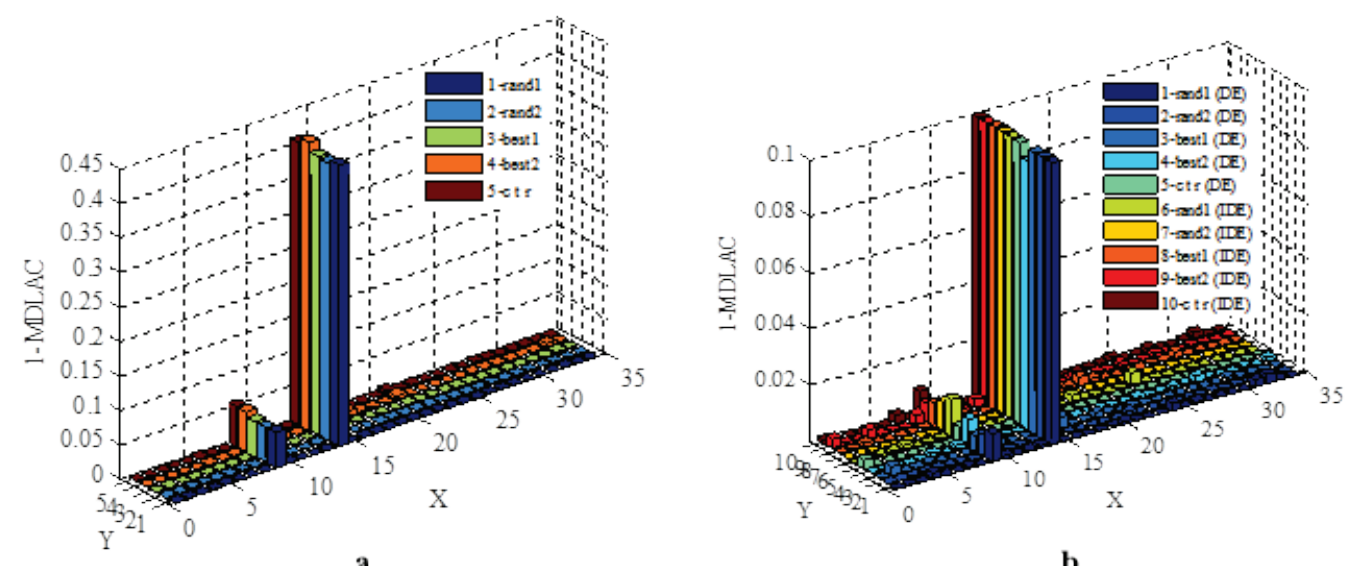

b

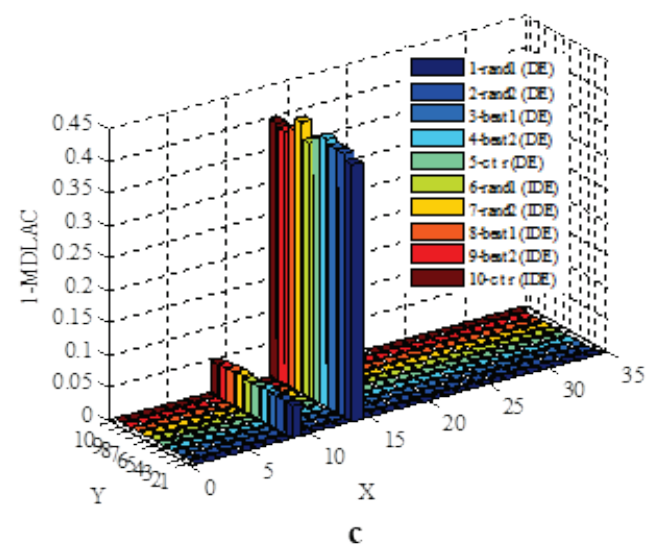

Fig. 5. Results of damage identification using multi-stage method for DE and IDE. (a) basic DE; (b) Stage 1 of present DE and IDE, (c) Stage 2 of present DE and IDE.

Table 3 Statistical results of damage identification with basic DE and present DE and IDE

\begin{tabular}{lcccccccccccc}
\hline \multirow{2}{*}{ Mutation strategy } & \multicolumn{4}{c}{ Basic DE } & \multicolumn{4}{c}{ DE (present) } & \multicolumn{4}{c}{ IDE (present) } \\
& NSA & $f$ & $x_{1}$ & $x_{2}$ & NSA & $f$ & $x_{1}$ & $x_{2}$ & NSA & $f$ & $x_{1}$ & $x_{2}$ \\
\hline rand1 & 11219 & 0.9228 & 0.084 & 0.347 & 4164 & 0.9243 & 0.062 & 0.387 & 4838 & 0.9288 & 0.051 & 0.388 \\
rand2 & 13527 & 0.9215 & 0.080 & 0.354 & 6879 & 0.9234 & 0.058 & 0.363 & 4847 & 0.9261 & 0.045 & 0.363 \\
best1 & 9479 & 0.9127 & 0.063 & 0.369 & 3266 & 0.9315 & 0.058 & 0.360 & 2944 & 0.9306 & 0.048 & 0.373 \\
best2 & 10636 & 0.9221 & 0.057 & 0.337 & 2801 & 0.9316 & 0.058 & 0.360 & 2321 & 0.9311 & 0.055 & 0.391 \\
c t r & 14190 & 0.9184 & 0.059 & 0.378 & 8879 & 0.9302 & 0.054 & 0.377 & 6134 & 0.9205 & 0.044 & 0.365 \\
\hline
\end{tabular}

where, $\mathrm{NSA}=$ average number of structural analysis; $f=$ the value of objective function $x_{1}=$ damage extent of element $9 ; x_{2}=$ damage extent of element 14 . 

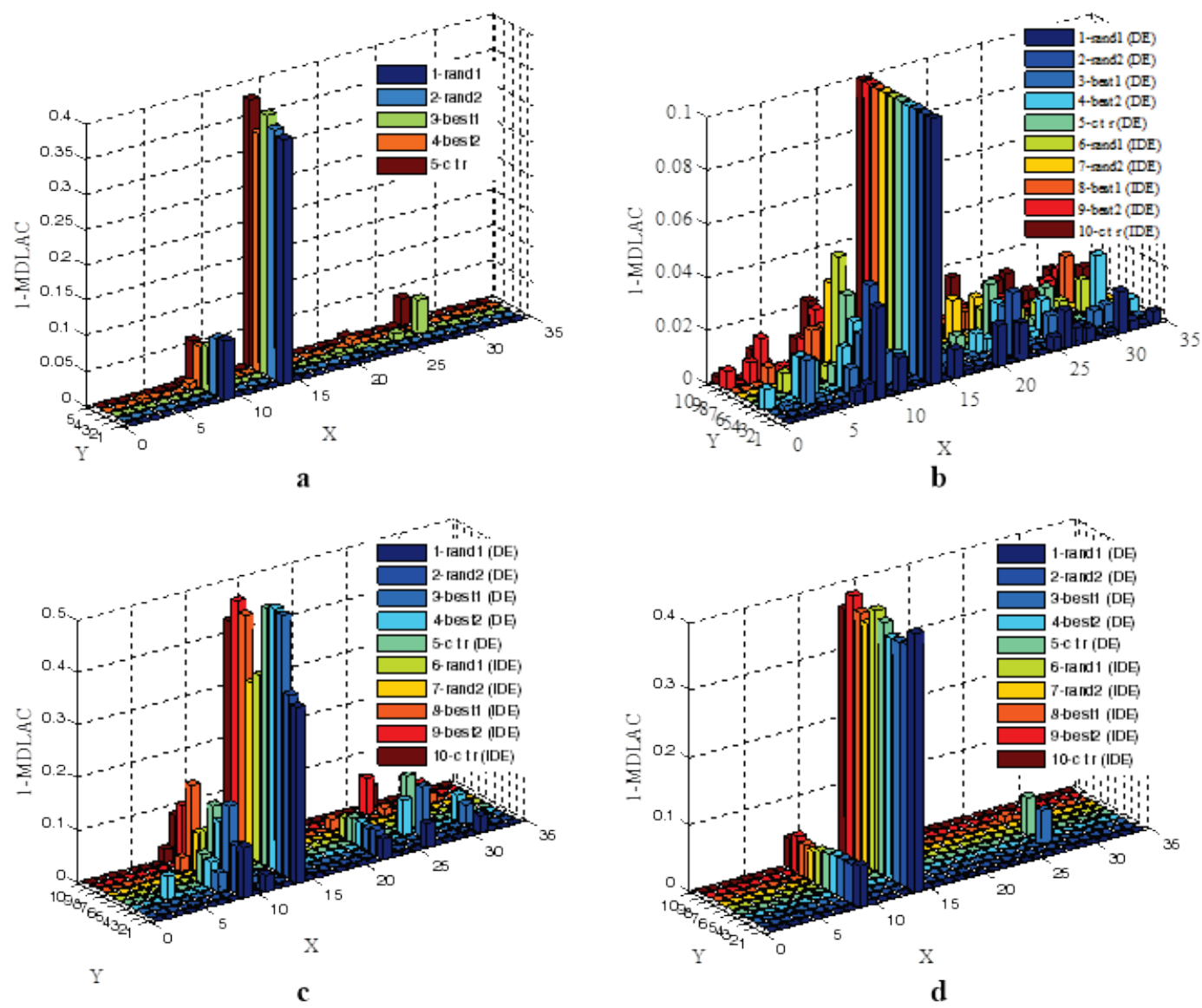

Fig. 6. Results of damage identification using basic DE and present DE and IDE. (a) basic DE; (b) Stage 1 of present DE and IDE, (c) Stage 2 of present DE and IDE; Stage 3 of present DE and IDE.

It can be seen from Figs. 6 (a) and (d), and Table 3, the relatively accurate locations and extents of damaged elements can be detected by both the basic DE and the present DE and IDE with three computational stages. However, for the computational accuracy, the present the DE and IDE is better than the basic DE, especially for the IDE with the more optimal solutions. For the computational cost, the NSA of the basic DE is more than the present DE and IDE, while the IDE with the five mutation strategies except the rand1 show the preferable performance than the present DE due the less NSA. Especially, it is worth mentioning that the best1 and best 2 are preferable due to the less NSA.

\subsection{A square composite plate}

\subsubsection{Model of damage}

The processes of multi-stage damage detection are also demonstrated using the laminated composite plate which is a three cross-ply $\left(0^{\circ} / 90^{\circ} / 0^{\circ}\right)$ clamped composite plate shown in Fig. 7. The parameters of material are as follows:

- Length of a side $a=1 \mathrm{~m}$,

- The thickness $t=0.1 \mathrm{~m}$,

- Young's Modulus $E_{1}=40 \mathrm{~N} / \mathrm{m}^{2}$,

- Young's Modulus $E_{2}=1 \mathrm{~N} / \mathrm{m}^{2}$,

- Poisson ratio $v_{12}=0.25$,

- Poisson ratio $v_{21}=0.00625$.

The free vibration behaviour of the composite plate was analysed in many literatures such as Ferreira et al. ${ }^{41}$ using a first-order shear deformation theory and T. VoDuy et al. ${ }^{28}$ using a two-step approach based on modal strain energy method and an improved differential evolution algorithm to detect the damage location and extent. In this example, the free vibration behaviour of the plate is obtained by the finte element method (FEM) using the ANSYS10.0, in which the plate is meshed 10 $\times 10$ of 8 -node shell99 element. Same as T. Vo-Duy et al. ${ }^{28}$, the two damage scenarios consisting of two and 
three damaged elements are considered in Table 4. The first eight frquencies of the plate in the healthy and damaged stage are presented in Table 5.

Table 4 Damage scenarios in the square composite plate

\begin{tabular}{cccc}
\hline \multicolumn{2}{c}{ Scenario 1 } & \multicolumn{2}{c}{ Scenario 2 } \\
\hline Element No. & Damage extent & Element No. & Damage extent \\
\hline 37 & 0.4 & 33 & 0.15 \\
47 & 0.6 & 57 & 0.2 \\
& & 74 & 0.25 \\
\hline
\end{tabular}

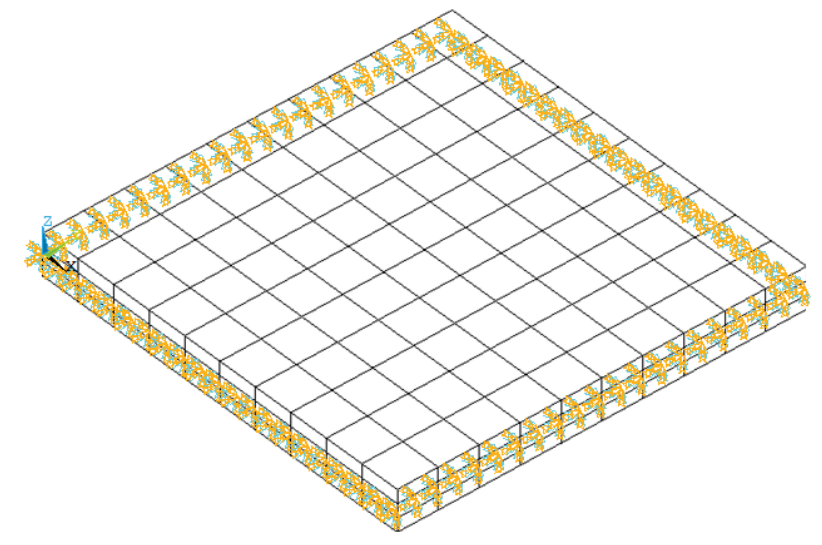

\subsubsection{Damage identification using the multi-stage method}

For both scenarios of damage, the MDLAC is computed by using the first ten frequencies and the first two mode shapes for the case of noise-free and the first ten frquencies and the first two, four, five and six mode shapes for the case of considering the measurement noise. It should be noted that the statistical results are the average solutions of ten times precedure and the average number of structural analysis (NSA). Besides, the stop criteria and the scaling factors are same with the section 5.1 both in cases of noise-free and noise $3 \%$.

Fig. 7. (a) A square composite plate discretized by the FEM; (b) element numbering of the composite plate.

Table 5 First eight frequencies of the healthy and damaged stage in the square composite plate

\begin{tabular}{ccccccc}
\hline Mode & $\begin{array}{c}\text { Intact } \\
\text { Ref. 28 }\end{array}$ & $\begin{array}{c}\text { Damaged } \\
\text { case 1 } \\
\text { Ref. 28 }\end{array}$ & $\begin{array}{c}\text { Damaged case 2 } \\
\text { Ref. 28 }\end{array}$ & $\begin{array}{c}\text { Intact } \\
\text { (Present) }\end{array}$ & $\begin{array}{c}\text { Damaged } \\
\text { case 1 } \\
\text { (Present) }\end{array}$ & $\begin{array}{c}\text { Damaged } \\
\text { case 2 } \\
\text { (Present) }\end{array}$ \\
\hline 1 & 7.444 & 7.377 & 7.422 & 7.415 & 7.393 & 7.410 \\
2 & 10.438 & 10.377 & 10.400 & 10.456 & 10.435 & 10.442 \\
3 & 13.990 & 13.850 & 13.951 & 13.947 & 13.888 & 13.928 \\
4 & 15.520 & 15.378 & 15.458 & 15.621 & 15.576 & 15.596 \\
5 & 15.887 & 15.813 & 15.841 & 15.926 & 15.902 & 15.906 \\
6 & 19.686 & 19.552 & 19.622 & 19.824 & 19.785 & 19.803 \\
7 & 21.627 & 21.022 & 21.482 & 21.556 & 21.541 & 21.550 \\
8 & 21.813 & 21.602 & 21.726 & 22.044 & 21.983 & 22.025 \\
\hline
\end{tabular}




\section{Case of noise free}

In the case of noise free, only the present methods of the mult-stage DE and IDE are used to detect the damage locations and extents. The statistical result of mutation strategie (best1) is shown in Fig. 8 and Table 6.

Fig. 8 inllustrates that three damaged elements are located and quantitated exactly for the case of noise free without any false alarm elements by using the present DE and IDE. Table 6 indicates that the locations and extents of damaged elements are in good agreement with the corresponding results obtained by Ref. 28 . However, it should be noted that the results of the NSA from Ref. 28 only include the quantification of damage severities without considering the computation amount of detecting the damage sites. It can be seen that the present IDE method with the new adaptive scaling factor is faster as compared to the present DE and the DE in Ref. 28, and that it isn't too slow than the IDE in Ref. 28 although it counts the all stages of determining the damage sites and extents relative to Ref. 28 .

2. Case of noise level $3 \%$

Figs. 9 and 10 provide the statistical results in term of average solutions and the average number of structural analyses for damage scenarios 1 and 2, respectively. The comparison of the present IDE using the different mode shapes and Ref. 28 is listed in Table 7.

As shown in Figs. 9, 10 and Table 7, the present IDE with 6 modes has better results in terms of accuracy than the present IDE with 2, 4, 5 modes and the DE in Ref. 28. Fig. 9 indicates that the false damage elements of element 32 using 2 and 4 modes and element 100 using 5 modes are detected. Fig. 10 illustrates that the false damage elements of element 58 and 62 are identified but the true damage element 57 is lost in Fig. 10 (a), and element 58 using 4 and 5 modes are wrongly detected in Figs. 10 (a) and (b). Therefore, it can be concluded that the more the number of the modes is, the higher the precision of identification is. For the computing time, the present IDE with different modes is faster than the DE in Ref. 28. However, due to the Ref. 28 only include the quantification of damage severities, the present IDE still shows superior computing performance.

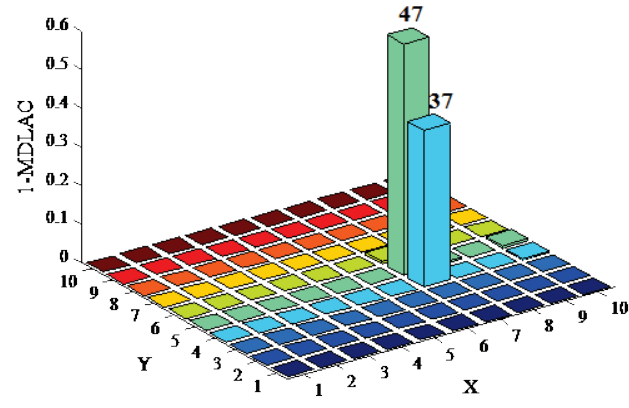

$\mathbf{a}$

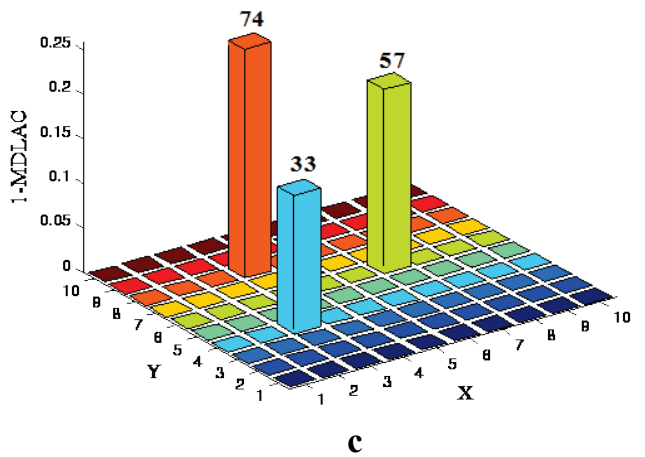

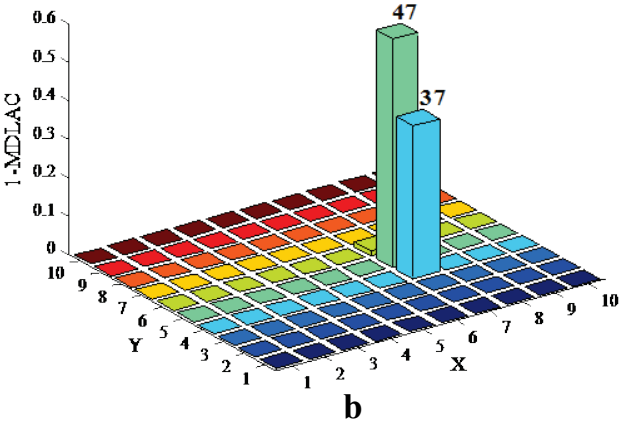

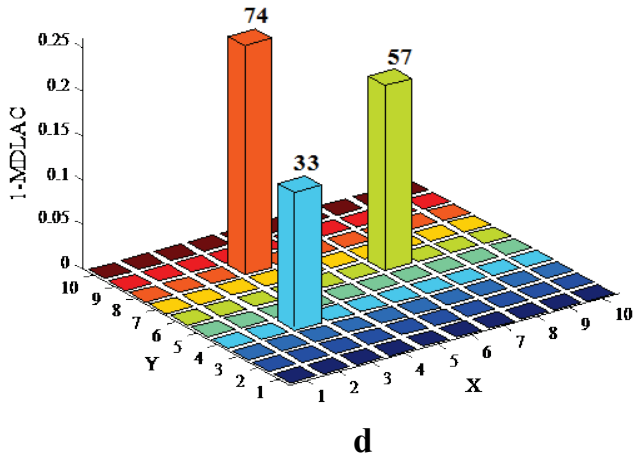

Fig. 8. Results of damage identification using the present DE and IDE without noise. (a) present DE of scenario 1; (b) present IDE of scenario 1; (c) present DE of scenario 2; (d) present IDE of scenario 2. 
Table 6 Statistical results of damage identification for the square composite plate in the case of noise-free

\begin{tabular}{lccccccccc}
\hline \multirow{2}{*}{ Mutation strategy } & \multicolumn{4}{c}{ Scenario 1 } & \multicolumn{4}{c}{ Scenario 2 } \\
& NSA & $f$ & $x_{1}$ & $x_{2}$ & NSA & $f$ & $x_{1}$ & $x_{2}$ & $x_{3}$ \\
\hline DE [28] & 7548 & $1.8519 \times 10^{-08}$ & 0.400 & 0.600 & 8872 & $6.3076 \times 10^{-07}$ & 0.150 & 0.200 & 0.250 \\
IDE [28] & 2660 & $4.3163 \times 10^{-07}$ & 0.400 & 0.600 & 2520 & $4.5083 \times 10^{-07}$ & 0.150 & 0.200 & 0.250 \\
DE (present) & 4174 & 0.999989 & 0.403 & 0.600 & 4788 & 0.999982 & 0.151 & 0.202 & 0.251 \\
IDE (present) & 3422 & 0.999982 & 0.398 & 0.599 & 3425 & 0.999993 & 0.151 & 0.201 & 0.252 \\
\hline
\end{tabular}

where, $\mathrm{NSA}=$ average number of structural analysis; $\mathrm{NS}=$ number of stages; $f=$ the value of objective function; for scenario 1 : $x_{1}=$ damage extent of element $37, x_{2}=$ damage extent of element 47 ; for scenario 2 : $x_{1}=$ damage extent of element $33, x_{2}=$ damage extent of element 57 and $x_{3}=$ damage extent of element 74 .
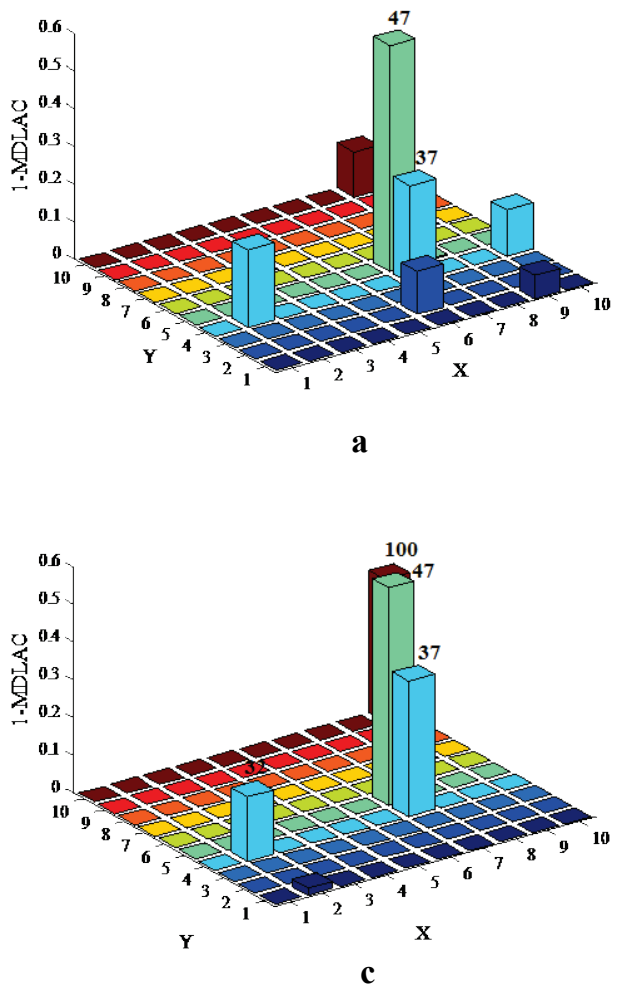

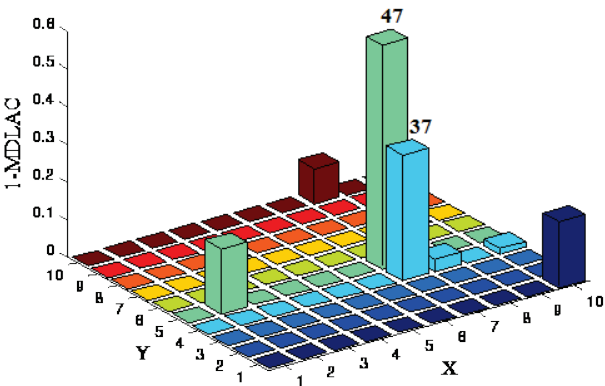

b

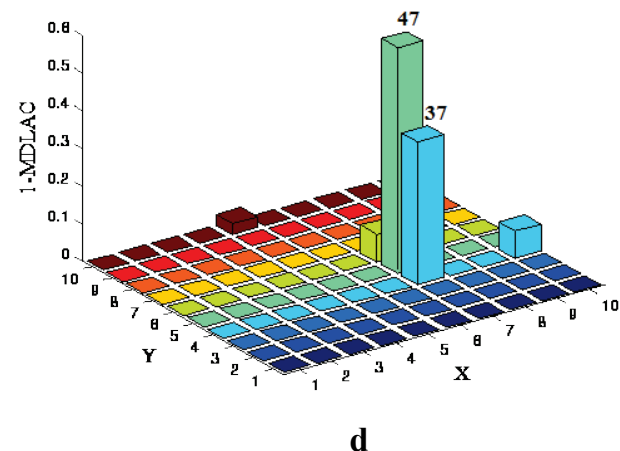

Fig. 9 Results of damage identification using the present IDE with noise 3\% for Scenario 1. (a) 2 mode shapes; (b) 4 mode shapes; (c) 5 mode shapes; (d) 6 mode shapes.

Table 7 Statistical results of damage identification for the square composite plate in the case of noise-free

\begin{tabular}{lccccccccc}
\hline \multicolumn{1}{c}{ Mutation strategy } & \multicolumn{3}{c}{ Scenario 1 } & \multicolumn{3}{c}{ Scenario 2 } \\
& NSA & $f$ & $x_{1}$ & $x_{2}$ & NSA & $f$ & $x_{1}$ & $x_{2}$ & $x_{3}$ \\
\hline DE [28] & 7644 & 0.1035 & 0.4055 & 0.5956 & 13956 & 0.1056 & 0.1457 & 0.1976 & 0.2511 \\
IDE [28] & 2688 & 0.1035 & 0.4053 & 0.5957 & 3464 & 0.1056 & 0.1458 & 0.1975 & 0.2511 \\
IDE (present (2 modes)) & 4008 & 0.8997 & 0.2574 & 0.6000 & 4851 & 0.8747 & 0.1133 & 0 & 0.1945 \\
IDE (present (4 modes)) & 4170 & 0.9002 & 0.3338 & 0.5955 & 4925 & 0.9107 & 0.1428 & 0.1391 & 0.2488 \\
IDE (present (5 modes)) & 3892 & 0.9045 & 0.3911 & 0.5971 & 4340 & 0.9221 & 0.1495 & 0.2009 & 0.2489 \\
IDE (present (6 modes)) & 4000 & 0.9054 & 0.3994 & 0.6000 & 4295 & 0.9162 & 0.1524 & 0.2024 & 0.2507 \\
\hline
\end{tabular}

where, $\mathrm{NSA}=$ average number of structural analysis; $\mathrm{NS}=$ number of stages; $\mathrm{f}=$ the value of objective function; for scenario 1 : $\mathrm{x} 1=$ damage extent of element $37, \mathrm{x} 2=$ damage extent of element 47 ; for scenario 2 : $\mathrm{x} 1=$ damage extent of element 33 , $\mathrm{x} 2=$ damage extent of element 57 and $\times 3=$ damage extent of element 74 . 

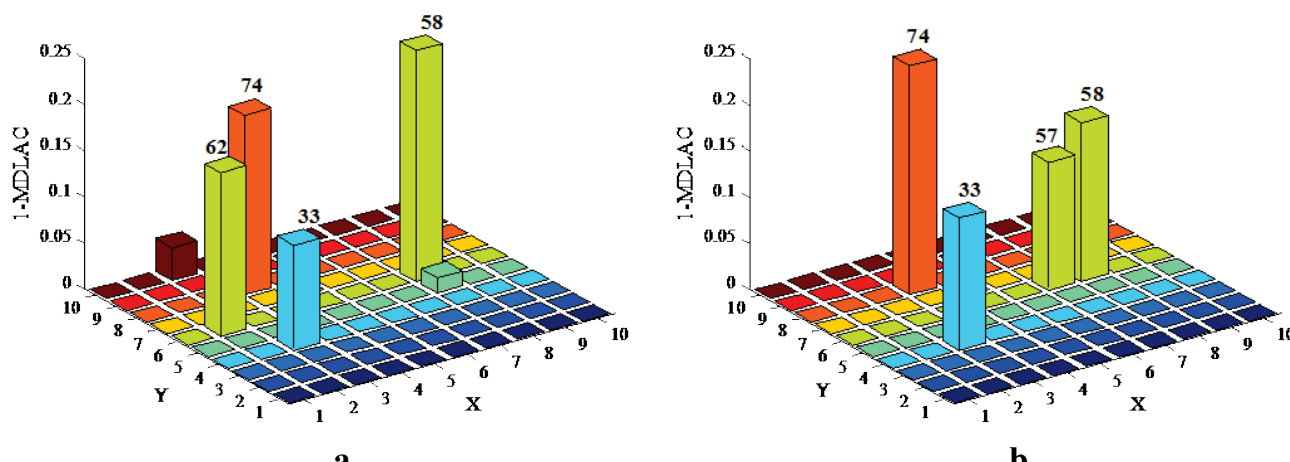

b
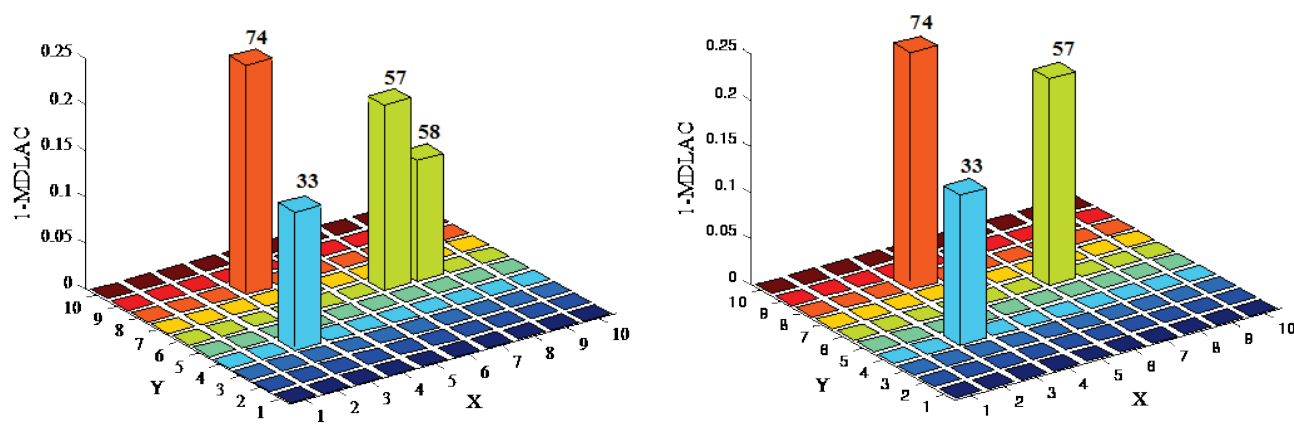

c

d

Fig. 10. Results of damage identification using the present IDE with noise 3\% for Scenario 2. (a) 2 mode shapes; (b) 4 mode shapes; (c) 5 mode shapes; (d) 6 mode shapes.

\section{Conclusions}

In this work, a multi-stage method based on multiple damage location assurance criterion and an improved differential evolution algorithm is presented for damage identification of civil engineering structures. The computational cost of damage identification process is reduced as far as possible by the suitable selection of damage range and filtering threshold in defferent computational stages. The IDE is proposed by a new adaptive scaling factor with a segmented function used in the mutaion operator of the basic differential evolution (DE) algorithm. The numerical examples for a continuous beam and a square composite plate are performed to show the effectiveness and robustness of the present method. In these examples, different damage scenarios are considered by reducing the stiffness of these elements as well as the effect of noise is investigated. The results demonstrated that the multistage method based on MDLAC and the IDE in each stage is effective for both locating and quantifying the damage regardless of the effect of noise. For computational accuracy and cost, the present IDE with more mode shapes has better performance than the basic DE and DE in Ref. 28.

\section{Acknowledgements}

This work was supported by the "985 Project" of Jilin University, the National High Technology Research and Development Program of China and the National Natural Science Foundation of China (Nos.51378236). The financial support is gratefully acknowledged.

\section{References}

1. Carden EP, Vibration Based Condition Monitoring: A Review. Struct Heal Monit, 3 (2004), pp. 355-77.

2. Wei Fan, Pizhong Qiao, Vibration-based Damage Identification Methods: A Review and Comparative Study, Struct Heal Monit, 10 (2011), pp. 83-111.

3. Fan W, Qiao P, Vibration-based damage identification methods: a review and comparative stud, Struct Heal Monit, 10(1) (2011), pp. 83-111.

4. Sohn H, Farrar CR, Hemez FM, Shunk DD, Stinemates DW, Nadler BR, A review of structural health monitoring literature: 1996-2001, Technical reports LA- 
13976-MS (Los Alamos National Laboratory, 2003).

5. Palacz M, Krawczuk M, Vibration parameters for damage detection in structures, J Sound Vib, 249(5) (2002), pp. 999-1010.

6. Kessler SS, Spearing SM, Atalla MJ, Cesnik CES, Soutis C, Damage detection in composite materials using frequency response methods, Compos Part B Eng, 33 (2002), pp. 87-95.

7. S.S. Naseralavi et al, Subset solving algorithm: A novel sensitivity-based method for damage detection of structures, Appl Math Model, 35 (2011), pp. 2232 2252.

8. Maeck, G. de Roeck. Damage assessment using vibration analysis on the Z24-bridge, Mech Syst Signal Pr, 17(2003), pp. 133-142.

9. Ndambi J-M, Vantomme J, Harri K, Damage assessment in reinforced concrete beams using eigenfrequencies and mode shape derivatives. Eng Struct 2002; 24:501-15.

10. F. Khoshnoudian, A. Esfandiari, Structural damage diagnosis using modal data, Scientia Iranica, Transactions A: Civil Engineering, 18 (2011), pp. 853 - 860.

11. Wei Chen et al, Damage Detection Based on Optimized Mode Shape and Frequency, Acta Mechanica Solida Sinica, 1(28), (2015).

12. Hamey CS, Lestari W, Qiao P, Song G, Experimental Damage Identification of Carbon/Epoxy Composite Beams Using Curvature Mode Shapes, Struct Heal Monit, 3 (2004), pp. 333-53.

13. Lestari, Qiao W and, Hanagud P and, Sathya, Curvature Mode Shape-based Damage Assessment of Carbon/Epoxy Composite Beams, J Intell Mater Syst Struct, 18 (2007), pp. 189-208.

14. A.Alvandi,C.Cremona,Assessment of vibration-based damage identification techniques, J Sound and Vib, 292 (2006), pp. 179-202.

15. S.M. Seyedpoor, O. Yazdanpanah, An efficient indicator for structural damage localization using the change of strain energy based on static noisy data, Appl Math Model, 38 (2014), pp. 2661-2672.

16. K.C. Park, G.W.Reich, K.F.Alvin, Structural damage detection using localized flexibilities, J Intel Mat Syst Str, 9 (1998), pp. 911-919.

17. L.T.Stutz, D.A.Castello, F.A.Rochinha, A flexibilitybased continuum damage identification approach, $J$ Sound Vib, 279(2005), pp. 641-667.

18. Li J, Dackermann U, $\mathrm{Xu}$ Y $\mathrm{L}$, et al, Damage identification in civil engineering structures utilizing PCA - compressed residual frequency response functions and neural network ensembles, Struct Control Hlth, 18(2) (2011), pp. 207-226.

19. Bandara R P, Chan T H T, Thambiratnam D P, Frequency response function based damage identification using principal component analysis and pattern recognition technique, Eng Struct, 66 (2014), pp. 116-128.

20. Shu J, Zhang Z, Gonzalez I, et al, The application of a damage detection method using Artificial Neural Network and train-induced vibrations on a simplified railway bridge model, Eng Struct, 52 (2013), pp. 408421.
21. Rajendra M, Shankar K, Improved Complex-valued Radial Basis Function (ICRBF) neural networks on multiple crack identification, Appl Soft Comput, 28 (2015), pp. 285-300.

22. Guo H Y, Li Z L, A two-stage method to identify structural damage sites and extents by using evidence theory and micro-search genetic algorithm, Mech Syst Signal Pr, 23(3) (2009), pp. 769-782.

23. Guo H Y, Li Z L, Structural damage identification based on Bayesian theory and improved immune genetic algorithm, Expert Sys Appl, 39(7) (2012), pp. 64266434.

24. Kang F, Li J, Xu Q, Damage detection based on improved particle swarm optimization using vibration data, Appl Soft Comput, 12(8) (2012), pp. 2329-2335.

25. Mohan S C, Maiti D K, Maity D. Structural damage assessment using FRF employing particle swarm optimization, Appl Math Comput, 219(20) (2013), pp. 10387-10400.

26. Friswell MI, Penny JET, Garvey SD, A combined genetic and eigensensitivity algorithm for the location of damage in structures, Comput Struct, 69 (1998), pp. 547-56.

27. Yang Z-B, Chen X-F, Xie Y, Miao H-H, Gao J-J, Qi K$Z$, Hybrid two-step method of damage detection for plate-like structures, Struct Control Heal Monit, 2015.

28. Vo-Duy, T., Ho-Huu, V., Dang-Trung, H., NguyenThoi, T., A two-step approach for damage detection in laminated composite structures using modal strain energy method and an improved differential evolution algorithm, Compos Struct, (2016).

29. H.M. Reedetal, A modified differential evolution algorithm for damage Identification in submerged shell structures, Mech Syst Signal Pr, 39(2013), pp. 396-408.

30. A. Rama Mohan Rao et al. Damage diagnostic technique for structural health monitoring using POD and self adaptive differential evolution algorithm, Comput Struct, 106-107 (2012), pp. 228-244.

31. Hou Z, Noori M, St Amand R. Wavelet-based approach for structural damage detection, $J$ Eng Mech, 2000, 126 (2000), pp.677-683.

32. Chang $\mathrm{C}$, Chen $\mathrm{L}$, Vibration damage detection of a Timoshenko beam by spatial wavelet based approach, Appl Acoust, 64 (2003), pp.1217-1240.

33. E. Grande, M.Imbimbo. A multi-stage approach for damage detection in structural systems based on flexibility, Mech Syst Signal Pr, 76-77 (2016) 455-475.

34. Williams E J , Messina A, Applications of the multiple damage location assurance criterion, Key Eng Mat, 167168 (1999), pp. 256-264.

35. B.H. Koh, S.J. Dyke, Structural health monitoring for flexible bridge structures using correlation and sensitivity of modal data, Comput Struct, 85 (2007), pp. 117-130.

36. H. Y. Guo, Z. L. Li. A two-stage method to identify structural damage sites and extents by using evidence theory and micro-search genetic algorithm. Mech Syst Signal Pr, 2009 (23): 769-782.

37. Wu Deng, Xinhua Yang, Li Zou, Meng Wang, Yaqing Liu, Yuanyuan Li, An improved self-adaptive 
differential evolution algorithm and its application, Chemometr Intel Lab, 128 (2013), pp. 66-76.

38. L.H. Wu, Y.N. Wang. Self-adapting control parameters modified differential evolution for trajectory planning of manipulator. J Control Theory App, 5 (4) (2007), pp. 365-373.

39. Qin AK, Huang VL, Suganthan PN. Differential evolution algorithm with strategy adaptation for global numerical optimization. IEEE Trans Evol Comput, 13(2)
(2009), pp. 398-417.

40. Abbass H, The self-adaptive Pareto differential evolution algorithm. In: Proceedings of the congress on evolutionary computing, 1 (2002). pp. 831-6.

41. Ferreira a. JM, Castro LMS, Bertoluzza S, A high order collocation method for the static and vibration analysis of composite plates using a first-order theory, Compos Struct, 89 (2009), pp. 424-32. 